\title{
Rehidratación en diarrea aguda con solución oral sodio 60
}

\author{
Dr, Gustavo Solar Y.1; Dr. Jose Ignacio Infante L ${ }_{r}^{2}$; Dra. Francisca Ugarte P. ${ }^{3}$ \\ Oral rehydratation in children with acute \\ diarrhea and mild to moderate dehydration
}

\begin{abstract}
A new oral rehydration solution (ORS) containing $\mathrm{Na}^{+} 60 \mathrm{mEq} / \mathrm{L} ; \mathrm{K}^{+} 20 \mathrm{mLq} / \mathrm{L} ; \mathrm{Cl}^{-} 50 \mathrm{mE}$; $/ \mathbf{L}$; citrate (as sodium citrate) $30 \mathrm{mLq} / \mathrm{L}$ and glucose $(25 \mathrm{~g} / \mathrm{L})$ was given to 30 otherwise normal children with acute diarrhea and mild to moderate dehydration. Another 22 infants matched for age and health status were given intravenous fluid therapy (IVS). Succesful hydration was achieved in both groups but the time to end point hydratation was shorter for ORS than for lVS (mean time 16.4 vs $24.6 \mathrm{~h}, \mathrm{p}=0.01$ ). No diferences were observed in the final serum . va ${ }^{+}$level but significantly higheI end serum $\mathrm{K}^{+}$concentration was obtained after ORS (ORS mean 4.2 ys $\Gamma \mathrm{V}$ mean 3.4 $\mathrm{mJiq} / \mathrm{L}$, p 0.001 ). End point arterial blood $\mathrm{pH}$ was higher and base excess lower in ORS (ORS pH mean 7.39 vs. IVS pH mean 7.33; $p=0.001$ and ORS BE mean -4.3 vs. $-7.9: p 0.005$ ). Only thee patients initially assigned to ORS were finally treated with IVS because of petsistent vomitus.

(Key words: orai hy dration, acute diarrhea).
\end{abstract}

E1 sindrome diarreico es una de las enfermedades mass comunes de la infancia; según datos de la O.M.S. en la mayoría de los países en desarrollo 20 a $30 \%$ de las defunciones de niños menores de cinco años son consecuencia de las enferme. dades diarreicas, lo que representa alrededor de cuatro a cinco millones de muertes anuales. ${ }^{1}$ Actualmente en Chile el riesgo de morir a causa de diarrea aguda ha disminuido notoriamente, pero esta disminución de la mortalidad no va aparejada con un descenso de la morbilidad la que sigue siendo elevada y causa frecuente de consulta y hospitalización especialmente en el período estival.

Uno de los objetivos básicos del tratamiento

1. Jefe Servicio Pediatría Hospital Exequiel González. Cortés.

2. Departamento de Pediatría y Cìrugía Infantil División Cs, Médicas Sur, Facultad de Medicina Universidad de Chile.

3. Becado Servicio y Departamento Pediatría, Hospital Exequiel González Cortés. de la diarrea aguda es la recuperación del contenido de agua y electrolitos, para lo cual se han usado diferentes vías de administración siendo la endovenosa la más difundida, no obstante las experiencias de Schultz y Zalusky ${ }^{2}$ quienes demostraron un aumento de la absorción de sodio en presencia de glucosa y posteriormente de Phillips $^{3}$ que de mostró que la absorción entérica de sodio y agua mediada por la glucosa, permanecia intacta en el cólera, permitiendo el uso de la vía oral en la rehidratación de la diarrea aguda, iniciándose en varios páíses el uso de la solución propuesta por la Organización Mundial de la Salud, ${ }^{4}$ en Chile solo se han realizado algunas experiencias con esta solución sin que su uso se haya difundido en forma masiva. ${ }^{5-8}$

Dado el predominio en nuestro medio de las diarreas no secretoras, en las cuales las concentraciones de sodio en las deposiciones son alrededor de $60-70 \mathrm{mEq} / \mathrm{L}^{9}$ el uso de soluciones con $90 \mathrm{mEq}$ de sodio podría inducir a hipernatremia, por esta razón en conjunto con el Laboratorio Chile hemos formulado una solución con $60 \mathrm{mEq}$ 
de sodio por litro y citrato como alcalinizante, manteniendo la proporción de los otros compo. nentes y una osmolaridad de $299 \mathrm{mM} / \mathrm{L}$. Esta solución fue administrada a un grupo de lactantes y los resultados obtenidos son el motivo de esta publicación.

\section{PACIENTES Y METODOS}

El estudio incluyó a lactantes entre 2 y 24 meses de edad, eutróficos 0 desnutridos leves, portadores de diarrea aguda con deshidratación leve o moderada, sin otra patología agregada y que no hubieran recibido hidratación anterior. Los pacjentes se hospitalizaron ent la unidad de lactantes del Servicio de Pediatría del Hospital Infantil Dr. Exequiel González Cortés durante el mes de Febrero de 1986 y se dividier on al azar en dos grupos: une recibió solución rehidratante oral por gastrocisis (30 pacientes) (HO) y un grupo control recibjó hidratación endovenosa ( 22 pacientes) (HEV).

La terapia oral consistió en la administración de la solución rehidratante oral, cuya composición es la siguiente glucosa $25 \mathrm{~g} / \mathrm{L}$; sodio $60 \mathrm{mEq} / \mathrm{L}$; potasio 20 $\mathrm{mEq} / \mathrm{L}$; cloro $S 0 \mathrm{mE}, \mathrm{L} / \mathrm{L}$; citrato de sodio $30 \mathrm{mEq} / \mathrm{L}$, en gastroclisis en volúmenes de $108 \mathrm{ml} \mathrm{x} \mathrm{kg} \mathrm{de} \mathrm{peso} \mathrm{las}$ primeras 6 horas, lo que equivale a 6 gotas $x \mathrm{~kg} . x$ minuto y $150 \mathrm{ml} \times \mathrm{kg}$ de peso las 18 horas restantes, repartídos en $120 \mathrm{ml}$ de solución y $30 \mathrm{ml} \mathrm{x} \mathrm{kg} \mathrm{de}$ alimentación láctea. El grupo contral recibió hidratación por yía endovenosa en base a solución glucosada al $5 \%$ en volúmenes de $180-200 \mathrm{ml} \times \mathrm{kg}$ de peso $\mathrm{x}$ día con agregado de cloruro de sodio al $10 \%$ según el tipo osmolar de deshidratación, cloruro de potasio al $10 \%$ $2-4 \mathrm{mEq} x \mathrm{~kg}$ de peso $y$ bicarbona to de sodio al $5 \%$ $2-4 \mathrm{mF}$. $\mathrm{x} \mathrm{kg}$ peso según grado de acidosis clínica.

A ambos grupos se les reakzar on evaluaciones de peso c hidratación a las $6-12-24-36 y$, ó, 48 horas y determinación de electrolitos plasmáticos y bases en sangro al ingreso y a las 24 y 48 horas. Se consideró fracaso de la hidratación oral la persistencia de vómitos a pesar de la reducción del goteo a 3 gotas $x \mathrm{~kg}$ de peso por minuto durante 30 minutos, aparición de crisis convulsivas e imposibjlidad de mejorar el estado de hidratación a las 6 horas.

\section{RESULTADOS}

Los volúmenes recibidos por los pacientes de ambos grupos se muestran en la tabla 1 y corresponden a los aportes iniciales y en 24 horas establecidos en el protocolo. La hidratación adecuada se alcanzó en promedio en forma más rápida en los pacientes sometidos a terapia de rehidratación oral lo que resultó estadísticamente significativo (p: 0,01) (tabla 2 ).

En los dos grupos el aumento de peso fue similar con cifras de $5,3 \%$ y $5,9 \%$ de aumento en los con terapia oral y endovenosa respectivamente. Las concentraciones iniciales de sodio plas-
Tabla 1.

Volümenes aportados ( $\mathrm{mL} \times \mathrm{kg}$ )

\begin{tabular}{|c|c|c|c|c|c|c|}
\hline & \multicolumn{3}{|c|}{ Vís oral } & \multicolumn{3}{|c|}{ Flebaclisis } \\
\hline & Min. & Prom. & Máx. & Min. & Prom. & Máx \\
\hline $\begin{array}{l}\text { Inivial } \\
\text { (6 horas) }\end{array}$ & 63 & 97 & 130 & . & & \\
\hline 24 holas & 154 & 216 & 285 & 170 & 193 & 200 \\
\hline
\end{tabular}

Tabla 2.

Tiempo de rehidratación (horas)

\begin{tabular}{|c|c|c|c|c|c|}
\hline \multicolumn{3}{|c|}{ Vía oral } & \multicolumn{3}{|c|}{ Flebocligis } \\
\hline Min. & Protn & Máx. & Min. & Prom. & Max. \\
\hline 6 & 16.4 & 36 & 6 & 24,6 & 18 \\
\hline
\end{tabular}

mátioo (HO: $\bar{x}$ 131, rango 123 a 144; HEV: $\bar{x}$ 134 , rango 122 a $140 \mathrm{mEq} / \mathrm{L} ; p=$ NS) no mostraron diferencias significativas con las concen. traciones finales (HO: $\bar{x} 130$, rango 115 a 142; $\mathrm{HEV}: \bar{x} 134$, rango 129 a $143 \mathrm{mEq} / \mathrm{L} ; \mathrm{p}=\mathrm{NS}$ ).

Las concentraciones plasmáticas de potasio fueron similares al ingreso (HO: $\bar{x}, 3,7$, rango 2,5 a 5,6 ; HEV: $\bar{x} 3,5$, rango 1,5 a $4,9 \mathrm{mEq} / \mathrm{L}$, $\mathrm{p}=$ N.S.) pero significativamente mayores en el grupo que recibió hidratación oral en la evaluación final (HO: $\bar{x} 4,2$, rango 3,3 a 5,7 vs HEV: $\bar{x}$ $3,4$, rango 1,7 a $4,0 \mathrm{mEq} / \mathrm{L}, \mathrm{p}<0,001)$.

Las variaciones de $\mathrm{pH}$ y del exceso de base en ambos grupos de pacientes muestran mejor corrección đel equilibrio ácido básico en los pacientes que recibieron terapia oral, diferencia estadisticamente significativa (tablas 3 y 4 ). La terapia oral fracasó en tres pacientes debido, en todos, a vómitos profusos persistentes, que no cedieron al disminuir el goteo de la gastroclisis.

\section{COMENTARIO}

Ambas terapias son útiles para corregir la deshidratación en pacientes con diarrea aguda. En nuestra experiencia el tiempo para alcanzar la hidratación adecuada fue menor en promedio con la terapia oral. Se observó una diferencia significativa entre los valores finales de potasio, pH y exceso de base, entre los pacientes tratados vía oral y los tratados por vía endovenosa, lo que podría explicarse por el uso de soluciones con componentes en cantidades fijas y en mayores 
Tabla 3.

Variaciones del pH

\begin{tabular}{|c|c|c|c|c|c|c|}
\hline & Min. & $\begin{array}{l}\text { Inicial } \\
\text { Prom. }\end{array}$ & Máx. & Min. & $\begin{array}{l}\text { Final } \\
\text { Prom. }\end{array}$ & Máx. \\
\hline Vía oral & 7,18 & 7,27 & $7.43 \longrightarrow$ & 7,30 & 7,39 & $7,48 \longrightarrow$ \\
\hline Fleboclisis & 7,07 & 7.24 & 7,46 & 7,22 & 7,33 & لנ \\
\hline
\end{tabular}

N.S. = No significativo

Tabla 4.

Variaciones del exceso de basc

\begin{tabular}{|c|c|c|c|c|c|c|}
\hline & Min. & $\begin{array}{l}\text { Inicial } \\
\text { Prom. }\end{array}$ & Máx. & Min. & $\begin{array}{l}\text { Final } \\
\text { Prom. }\end{array}$ & Máx. \\
\hline Vía oral & $-17,5$ & $-11,2$ & $-2.7-$ & $-11,0$ & $-4,3$ & $+3 \longrightarrow 2005$ \\
\hline Flcboclisis & $-20,0$ & $-12,08$ & $+0.5 \stackrel{p N_{i}}{ }$ & $-16,7$ & $-7,93$ & 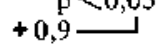 \\
\hline
\end{tabular}

N.S. = No significativo

concentraciones, que las utilizadas en los pacientes con fleboclisis, no observándose con ninguno de los dos esquemas la aparición de hipernatremia.

En nuestra opinión la terapia de rehidratación oral con una solución con $60 \mathrm{mEq} \times \mathrm{L}$ de sodio permite una recuperación rápida del trastorno hidroelectrolítico y ácido básico, con las ventajas ya sefialadas en comparación con la terapia endo. venosa $y$ al mismo tiempo elimina el factor de riesgo en relación a infecciones intrahospitalarias, que significa la administración de la terapia endovenosa, motivo por el cual recomendamos su uso en el tratamiento de rehidratación de la diarrea aguda con exclusión de los casos con deshidratación grave, presencia de shock, compromiso de conciencia o convulsiones e ileo paralítico. Esta modalidad de terapia sugerida ha sido adoptada cono norma del Servicio de Pediatría del Hospj. tal Exequiel González Cortés.

\section{RESUMEN}

Se compara la terapia de relidratación oral con el tratamient o endovenoso tradicional, mediante el uso de una solución especialmente formulada, con una concentración de $60 \mathrm{mEq} / \mathrm{L}$ de sodio y citrato como alcalinizante, en dos grupos de lactantes hospitalizados con diarrea aguda y deshidratación leve. La hidratación oral demostró ser más rápida en la corrección del desequilibrio hidroetectrolítico, así como en la mejor corrección del potasio y del trastorno ácido básico lo que fue estadisticamente signifi- cativo. En ambos grupos se obtuvo una hidratación adecuada final y no se observó la aparición de hipematremias. En el grupo con solución oral se presentaron tres fallas de tratamiento debido a vómitos incoercibles a pesar de disminuir durante media hora a la mitad la velocidad de la infusión. Por Jos buenos resultados obtenidos recomendamos ampliar al máximo el empleo de hidratación oral para el tratamiento de las deshidrataciones leves y moderadas en niños con diarrea aguda.

\section{REFERENCIAS}

1. WHO Programme for control of diarroheal aisease. "Fourth Programme Report 1983-1984".

2. Shutz S., Zalusky R: Ion transport in isolated rabbit ileum. The intercation between active sodium and sugar transport. J Gen Physiol 1964; 47: 1043.

3. Phillps R.A.: Water and electrolyte looses in cholcra. Fed Proc 1964; 23: 705-712.

4. WHO. A manual for the treatment of acure diarrhoea geneva. Programme for control of Diarrhoeal Discases. WHO/CDD/SER 80.2. 1980.

5. Cordero $P$. Araya $M$. . Espinoza $I$., Figuerod $G_{*}$, Pacheco I., Brinser P.: Efecto de la hidratación oral y realimentación precoz en la evolución de la diarrea aguda del lactante, Rev Chil Pediatr 1985; 56; 412-418.

6. Coichac A., Avilés A., Romero I., Pinto G.: Rehidratación oral en lactantes con diarrea aguda. Rev Chil Pediatr 1985; 56; 162-164.

7. Duffat G., Emilfork $M$., Donoso $F$., Aguirre M., Voldés $I$, Paredes $M$.: Rehidratación del lactante hospitalizado por diarrea aguda con solución oral OMS. Pediatria (Santiago) $1983 ; 26: 79-82$.

8. Emilfork $M$., Duffau G.: Evaluación de una solución oral en la terapia de mantenimiento del síndrome diarreico agudo sin deshidratación del lactante. Bol Med Hosp Infant Mex 1982; 39: 729-736. 